\title{
Identification of novel enriched recurrent chimeric COL7A1-UCN2 in human laryngeal cancer samples using deep sequencing
}

Ye Tao ${ }^{1 \dagger}$, Neil Gross ${ }^{2}$, Xiaojiao Fan ${ }^{3+}$, Jianming Yang ${ }^{4+}$, Maikun Teng ${ }^{3}, \mathrm{Xu} \mathrm{Li}^{3}$, Guojun $\mathrm{Li}^{2}$, Yang Zhang ${ }^{1 *}$ and Zhigang Huang ${ }^{1 *}$

\begin{abstract}
Background: As hybrid RNAs, transcription-induced chimeras (TICs) may have tumor-promoting properties, and some specific chimeras have become important diagnostic markers and therapeutic targets for cancer.

Methods: We examined 23 paired laryngeal cancer (LC) tissues and adjacent normal mucous membrane tissue samples (ANMMTs). Three of these pairs were used for comparative transcriptomic analysis using high-throughput sequencing. Furthermore, we used real-time polymerase chain reaction (RT-PCR) for further validation in 20 samples. The Kaplan-Meier method and Cox regression model were used for the survival analysis.

Results: We identified 87 tumor-related TICS and found that COL7AT-UCN2 had the highest frequency in LC tissues $(13 / 23 ; 56.5 \%)$, whereas none of the ANMMTs were positive $(0 / 23 ; p<0.0001)$. COL7A1-UCN2, generated via alternative splicing in LC tissue cancer cells, had disrupted coding regions, but it down-regulated the mRNA expression of COL7A1 and UCN2. Both COL7A1 and UCN2 were down-expressed in LC tissues as compared to their paired ANMMTs. The COL7A1: $\beta$-actin ratio in COL7A1-UCN2-positive LC samples was significantly lower than that in COL7A1-UCN2-negative samples $(p=0.019)$. Likewise, the UCN2: $\beta$-actin ratio was also decreased $(p=0.21)$. Furthermore, COL7A1-UCN2 positivity was significantly associated with the overall survival of LC patients ( $p=0.032 ; \mathrm{HR}, 13.2[95 \% \mathrm{Cl}, 1.2-149.5])$.

Conclusion: LC cells were enriched in the recurrent chimera COL7A1-UCN2, which potentially affected cancer stem cell transition, promoted epithelial-mesenchymal transition in LC, and resulted in poorer prognoses.
\end{abstract}

Keywords: Laryngeal cancer, Transcription-induced chimera, Gene fusion, COL7A1, UCN2

\section{Background}

There were an estimated 26,400 new cases of and 3620 deaths from laryngeal cancer in China in 2015 [1]. Like other carcinomas of the respiratory system, carcinogen exposure via tobacco smoke causes DNA damage, and the accumulation of this DNA damage can alter genetic and epigenetic regulatory functions and thereby transform normal cells into cancer cells $[2,3]$. This cell transformation usually takes multiple steps to complete, and it is affected by the sensitivity of the individual and the degree of damage [4]. This process is called tumorigenesis [5].

\footnotetext{
*Correspondence: zhangyangent@163.com; huangzhigang1963@sohu.com ${ }^{\dagger}$ Equal contributors

${ }^{1}$ Department of Otolaryngology-Head and Neck Surgery, Key Laboratory of Otolaryngology Head and Neck Surgery, Beijing Tongren Hospital, Capital Medical University, Beijing 100730, China

Full list of author information is available at the end of the article
}

Tumorigenesis often presents with chromosomal and DNA abnormalities, and one common chromosomal rearrangement is gene fusion [6]. Some specific gene fusions have become important diagnostic markers of and therapeutic targets in cancer over the past several decades [7]. These chimeric products are often associated with neoplastic behavior $[7,8]$. Typically, the $B C R$ $A B L 1$ fusion gene is rearranged via the $\mathrm{t}(8 ; 14)(\mathrm{q} 24 ; \mathrm{q} 32)$ translocation in Burkitt lymphoma cells. This rearrangement is caused by this gene's juxtaposition with regulatory elements of the immunoglobulin heavy chain gene at $14 \mathrm{q} 32$, where the $M Y C$ gene is constitutively activated due to its expression, which is driven by immunoglobulin enhancers $[7,9]$. Other fusion genes, including $P R C C$ TFE3 in papillary renal cell carcinoma [10], PAX8-PPARG in follicular thyroid carcinoma [11], FUS-CREB3L2 in soft 
tissue sarcoma [12], and TMPRSS2-ETS in prostate cancer [13], have gradually been identified with various potential gene regulation mechanisms.

As in the fusion of two DNA genes, the two adjacent RNA genes, which are in the same orientation and are usually transcribed independently, are occasionally transcribed into a single fused RNA sequence. The various splicing mechanisms involved in such a transcription include RNA editing, alternative splicing (AS), trans-splicing, alternative transcription start sites, and alternative polyadenylation transcription termination sites [14-17]. This single fused RNA sequence is called a transcriptioninduced chimera (TIC) [14]. Unlike a single transcript that can be translated into various proteins in prokaryotes, TICs usually do not produce chimeric proteins or independent transcripts. Instead, they have tumor-promoting properties as hybrid RNAs [14]. For example, the expression of the chimeric transcript $H B x-L I N E 1$ was associated with hepatocellular carcinoma development and correlated with poor survival [18]. Also, the chimeric transcript SLC45A3-ELK4, generated by cis-splicing between the adjacent $S L C 45 A 3$ and ELK4 genes, did not involve DNA rearrangements or trans-splicing and could augment prostate cancer cell proliferation [19].

In comprehensively analyzing novel TICs in transcriptomes in LC cells using a paired-end strategy for RNA deep sequencing, we found that COL7A1-urocortin 2 (UCN2) is a novel TIC. We could not elucidate the intrinsic genetic and epigenetic mechanism responsible for COL7A1-UCN2 generation; however, both the COL7A1 and UCN2 genes had explicit suppressor roles in tumor regulation, specifically the regulation of the epithelial-mesenchymal transition (EMT) [20-22]. Therefore, we hypothesized that COL7A1$U C N 2$ may down-regulate the mRNA expression of both COL7A1 and UCN2 in LC tissues and that such downregulation may promote tumor invasion via EMT regulation. Furthermore, we also speculate that COL7A1-UCN2 generation can reflect the degree of DNA damages and that this TIC positivity may be associated with LC prognosis.

\section{Methods}

\section{Patients and tissue samples}

The Institutional Review Board approval for this laryngeal cancer research project (No. TRECKY 2009-33; Date: Jan, 2009) was obtained from the Beijing Tongren Hospital of Capital Medical University. A total of 23 patients who underwent surgery for pathologically confirmed LC from 2009 to 2016 were enrolled in this study. All patients received and signed a written informed consent. These patients had archived tumor specimens and data available, with a minimum of 36 months of cancer-free or censored-death follow-up after surgery. The follow up was completed through monitoring of their medical records or conducting telephone interviews. To confirm the diagnosis, the tumors' histological classifications and differentiation were defined based on the 1999 World Health Organization's histological classification standards for LC. Tumor staging was carried out using the 2009 TNM staging criteria of the Union for International Cancer Control. Clinicopathological data were available for all 23 patients (Table 1).

All tumor samples contained more than $50 \%$ tumor cells and were stored at $-80{ }^{\circ} \mathrm{C}$ until use. Paired LC and adjacent normal mucous membrane tissue samples (ANMMTs) were obtained from the 23 patients. Paired samples from three male patients with T4N2aM0 disease and various degrees of differentiation (well, moderately, and poorly differentiated) who were 61-63 years old, smokers, and alcohol drinkers and had undergone total laryngectomy with selective bilateral neck dissection and

Table 1 Correlation of COL7A1-UCN2 expression with LC clinical characteristics

\begin{tabular}{|c|c|c|c|c|}
\hline \multirow[t]{3}{*}{ Characteristics } & \multirow{3}{*}{$\begin{array}{l}\text { Cases } \\
(\%)\end{array}$} & \multicolumn{2}{|c|}{ COL7A1-UCN2 mRNA expression } & \multirow{3}{*}{$p$} \\
\hline & & mRNA positive $(+)$ & mRNA negative $(-)$ & \\
\hline & & $N, \%$ & $\mathrm{~N}, \%$ & \\
\hline Age (years) & & & & 0.685 \\
\hline$\geq 60$ & $15(65.2)$ & $9(39.1)$ & $6(26.1)$ & \\
\hline$<60$ & $8(34.8)$ & $4(17.4)$ & $4(17.4)$ & \\
\hline Gender & & & & 1.000 \\
\hline Male & $20(87.0)$ & $11(47.8)$ & $9(39.1)$ & \\
\hline Female & $3(13.0)$ & $2(8.7)$ & $1(4.3)$ & \\
\hline Tumor stage & & & & 0.221 \\
\hline 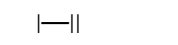 & $8(34.8)$ & $3(13.0)$ & $5(21.7)$ & \\
\hline$\| \mathrm{IIV}$ & $15(65.2)$ & $10(43.5)$ & $5(21.7)$ & \\
\hline Differentiation & & & & 1.000 \\
\hline Well & $4(17.4)$ & $2(8.7)$ & $2(8.7)$ & \\
\hline Moderate & $16(69.6)$ & $9(39.1)$ & $7(30.4)$ & \\
\hline Poor & $3(13.0)$ & $2(8.7)$ & $1(4.3)$ & \\
\hline LNM & & & & 0.685 \\
\hline No & $10(43.5)$ & $5(15.6)$ & $5(15.6)$ & \\
\hline $\mathrm{N}+$ & $13(56.5)$ & $8(34.8)$ & $5(15.6)$ & \\
\hline Treatment & & & & 0.685 \\
\hline S only & $8(34.8)$ & $4(17.4)$ & $4(17.4)$ & \\
\hline$S+C / X$ & $15(65.2)$ & $9(39.1)$ & $6(26.1)$ & \\
\hline Smoking & & & & 0.435 \\
\hline Ever & $22(95.7)$ & $13(56.5)$ & $9(39.1)$ & \\
\hline Never & $1(4.3)$ & $0(0.00)$ & $1(4.3)$ & \\
\hline Alcohol & & & & 1.000 \\
\hline Ever & 21 (91.3) & $12(37.5)$ & $9(39.1)$ & \\
\hline Never & $2(8.7)$ & $1(4.3)$ & $1(4.3)$ & \\
\hline
\end{tabular}

LNM lymph node metastasis, + positive, $C$ chemotherapy, $X$ radiation, $S$ surgery 
without preoperative chemotherapy or radiotherapy were prepared for transcriptomic analysis. The paired samples from the remaining 20 patients were used to validate the TIC using real-time polymerase chain reaction (RT-PCR). Adjacent normal tissue samples were obtained at least $5 \mathrm{~mm}$ from the tumor margins [23].

\section{Pathological review}

Slides with hematoxylin and eosin staining were used to contain the paired frozen tumor and normal tissue sections. These slides were subjected to pathological examination twice to ensure that tumor tissues carrying high-density cancer foci $(>75 \%)$ were used and that the normal tissue samples had no tumor components. All samples were examined and reviewed by two pathologists independently, and disagreements between them were resolved via negotiation.

\section{Preparation and sequencing of cDNA library}

The total RNA was isolated from the fresh tissues using TRIzol reagent (Sigma-Aldrich, Missouri, St. Louis, US) according to the manufacturer's instructions. Poly(A) mRNA was isolated from the total RNA using beads containing oligo(dT). A fragmentation buffer was used to fragment the purified mRNA. Using these short mRNA fragments as templates, random hexamer primers were applied to synthesize first-strand cDNA. The fragmentation buffer, RNase $\mathrm{H}$, and DNA polymerase I were used to synthesize the second-strand cDNA. Short double-stranded cDNA fragments were purified using a QIA quick PCR extraction kit (Qiagen, Hilden, Germany) and eluted with EB buffer for end repair and the addition of an "A" base. The short fragments were then ligated to Illumina sequencing adaptors (San Diego, CA, U.S.A.). DNA fragments of a selected size were gel-purified and amplified using PCR. The amplified library of fragments was sequenced using an Illumina HiSeq 4000 sequencing machine.

\section{Raw read filtering}

The images of the nucleotides generated by the Illumina HiSeq 4000 sequencing machine were converted into nucleotide sequences using a base-calling pipeline. The raw reads of the nucleotide sequences were saved in FASTQ format. The dirty raw reads were removed before the data analysis. Three removal criteria were used in filtering out dirty raw reads: 1 ) reads with sequence adapters, 2) reads with more than $2 \%$ "N" bases, and 3 ) low-quality reads. This ensured that clean reads were used for the subsequent mapping to the human genome and transcriptome.
Reads mapped to the human genome and transcriptome The Burrows-Wheeler Aligner software program was used to map clean reads to a reference genome, and the Bowtie software program was used to map them to a reference gene. The expression level of each gene was measured via the number of specific fragment reads mapped per kilobase exon model per million reads (RPKM). The formula used for mapping is as follows: $\mathrm{RPKM}=\frac{10^{9} \mathrm{C}}{N L}$. In this formula, $C$ stands for the number of fragments specifically mapped to a given gene, $N$ stands for the number of fragments specifically mapped to all genes, and $L$ stands for the overall length of exons for the given gene. For genes with more than one alternative transcript, the longest transcript was chosen for the calculation of the RPKM. The RPKM calculation avoids the effect of differing gene lengths and sequencing discrepancies. Thus, the differences in the gene's expressions between samples were directly compared using the RPKM.

\section{Differentially expressed gene analysis}

Differentially expressed genes were identified in the tumor and matched normal tissue samples according to two criteria - a false-discovery rate no greater than 0.001 and a $\log 2$ ratio of at least 1 . This approach was chosen based on the significance of digital gene expression profiles.

\section{Fusion of human gene detection}

During the read alignment of the short RNA and the reference genome, when the reads were divided into two fragments, only some of them could be aligned. Twosegment alignments could be read to the reference genome using the gene fusion-detection doctrines of the SOAPfuse software program, which can detect gene fusions using span and junction reads [24]. This basic method includes 1) comparing the reads to the reference genome alignment and the transcripts to the notes; 2) using the local genome library, which contains an exhaustive algorithm, to construct the fusion site sequence; and 3) retaining highly credible fusion transcripts using a series of filtering means. The requirements for the alignment detection of the divided reads were as follows: a length of at least $8 \mathrm{bp}$ for the shorter read segment and an intron boundary within one of the three canonical bounds (GT-AG, GC-AG, and AT-AC). Regardless of where the intron was derived, the boundaries always should be the same. For the DNA positive strand, for both read segment alignments, a maximum of one mismatch and an unmapped alignment was required. Based on the information on the alignments of the two segments, gene fusion sites identified from the mapping of the human genome and transcriptome were retrieved using a Perl script. A fused gene certainly existed if the fusion site was located at the known exon boundaries 
of the two genes, with at least one paired-end read supporting it [25-27].

\section{Detection of alternative splicing (AS)}

AS is a fundamental mechanism of the generation of transcript diversity. The base-calling pipeline used in this study to detect AS events in the transcriptome cDNA library consisted of two major steps. 1) SOAPsplice (Version 1.1) was used to map the reads to the human reference sequence and report the splice junctions according to the junction reads of the alignments [24]. With SOAPsplice, the default parameters were used as much as possible; three mismatches were set for intact alignments, and no more than one mismatch was set for splicing alignments. 2) Abased on AS mechanisms, both the junctions of splicing [e.g., known splice junctions obtained from the National Center for Biotechnology Information RefSeq database (Bethesda, MD, US)] and the results derived from the mapping were applied for the detection of the four basic AS events: the skipping of exons, sites of alternative $5^{\prime}$ splicing, sites of alternative 3 ' splicing, and the retention of introns.

By detecting the four types of AS events, those that occurred in the tumors, rather than in the matched normal tissue, were detected as specifically tumor-related AS events. The AS events that were detected in both LC and ANMMT samples were then filtered. Finally, for each sample, a list of highly reliable tumor-specific AS events was generated.

\section{Validation of transcriptome cDNA library using RT-PCR}

To determine the frequency of COL7A1-UCN2 and COL7A1 and UCN2 mRNA expression, the other 20 paired LC and ANMMT samples were subjected to RT-PCR analysis. The primer sequences used for this RT-PCR are listed in Table 2.

For the CDNA of COL7A1-UCN2 and COL7A1, the PCR conditions were $10 \mathrm{~min}$ at $95{ }^{\circ} \mathrm{C}, 30$ cycles of $30 \mathrm{~s}$ at $95{ }^{\circ} \mathrm{C}, 30 \mathrm{~s}$ at $62{ }^{\circ} \mathrm{C}, 90 \mathrm{~s}$ at $72{ }^{\circ} \mathrm{C}$, and $10 \mathrm{~min}$ at $72^{\circ}$ C. For UCN2 cDNA, the PCR conditions were $10 \mathrm{~min}$ at $95{ }^{\circ} \mathrm{C}, 30$ cycles of $30 \mathrm{~s}$ at $95^{\circ} \mathrm{C}, 30 \mathrm{~s}$ at $70{ }^{\circ} \mathrm{C}, 30 \mathrm{~s}$ at

Table 2 Primer sequences used for RT-PCR in the study

\begin{tabular}{ll}
\hline Primers & Sequences \\
\hline COL7A1-UCN2 & F: 5'-CGCCAAGAGATGAGTCAGCAC-3' \\
R: 5'-GCACTCAGATCTGATATGACCTGC-3' & F:5'-CGCCAAGAGATGAGTCAGCAC-3' \\
R:5'51 & F:-CTCTGCAGGTAGGGCAGGGT-3' \\
& R: 5'-TCAGCAGTGGCCGACACG-3' \\
UCN2 & F:5'-TTGCCGACAGGATGCAGAA-3' \\
B-actin & R:5'-GCCGATCCACACGGAGTACTT-3' \\
&
\end{tabular}

$72{ }^{\circ} \mathrm{C}$, and $10 \mathrm{~min}$ at $72{ }^{\circ} \mathrm{C}$. $\beta$-actin was used as a loading control. The RT-PCR products were analyzed using gel electrophoresis.

Quantitative analysis of PCR products was carried out using a Rotor-Gene 3000 (Corbett Research, Sydney, Australia) and a commercially available SYBR Premix Ex Taq Perfect Real-Time Kit (Takara Biotechnology, Dalian, China), which were used according to the manufacturer's instructions. The primer sequences used were those described above. The PCR conditions were $30 \mathrm{~s}$ at $95{ }^{\circ} \mathrm{C}, 40$ cycles of $5 \mathrm{~s}$ at $95^{\circ} \mathrm{C}$, and $30 \mathrm{~s}$ at $60{ }^{\circ} \mathrm{C}$. The data were analyzed using the $\Delta \Delta \mathrm{Ct}$ method, and values were expressed as the fold difference from the housekeeping gene, $\beta$-actin.

\section{Statistical analysis}

Data were expressed as means \pm standard deviation. Differences between the two groups were examined using Fisher's exact test (two-sided, $n<40$ ) or a paired or unpaired Mann-Whitney $U$-test. The Kaplan-Meier method and Cox regression model were used to perform the overall survival analysis of the 23 patients, who were grouped according to their positivity or negativity for COL7A1-UCN2. P-values less than 0.05 were considered statistically significant. The data were analyzed using the SPSS 20.0 statistical software program (IBM Corporation, Armonk, NY, USA).

\section{Results}

Transcriptome sequences in human LC and ANMMT samples

We compared the transcriptome sequences in LC and paired normal tissue samples and identified a series of gene fusions and differentially expressed genes. The RNA sequencing data for the three pairs of LC and ANMMT samples subjected to transcriptomic analysis are listed in Table 3.

\section{Landscapes of the TIC genome in LC tissues}

In the comparative transcriptome analysis of the three paired LC and ANMMT samples with distinct patterns of tumor differentiation, we identified 87 TICs. We detected the novel chimeric transcript fusion COL7A1UCN2 in two of the three LC samples but not in their paired ANMMT samples. Also, we did find a coding frameshift in this TIC (Fig. 1 and Additional file 1: Figure S1; Table 4).

Both the COL7A1 and UCN2 genes are located at 3p21.3. In COL7A1-UCN2, COL7A1 is located at exons 113-117 (from Chr. 3: 48602216 to Chr. 3: 48603724) and is $587 \mathrm{nt}$ long. UCN2 is located at exon 2 (from Chr. 3: 48600032 to Chr. 3:48600569) and is 538 nt long. In COL7A1-UCN2, the exon 2 sequence of UCN2 was 
Table 3 RNA sequencing data for three pairs of LC and ANMMT samples for transcriptomic analysis

\begin{tabular}{|c|c|c|c|c|c|c|c|c|c|}
\hline \multicolumn{2}{|c|}{ Sample } & \multirow{2}{*}{$\begin{array}{l}\text { Clean } \\
\text { reads }\end{array}$} & \multirow{2}{*}{$\begin{array}{l}\text { Expressed } \\
\text { genes }\end{array}$} & \multicolumn{2}{|c|}{ Expressed Transcripts } & \multicolumn{2}{|c|}{ Chimeric Transcripts } & \multirow{2}{*}{$\begin{array}{l}\text { Alternative splicing } \\
\text { Number }\end{array}$} & \multirow[t]{2}{*}{ SNP } \\
\hline ID & Tissue & & & Number & Novel- Transcripts & Number & COL7A1-UCN2 TIC & & \\
\hline \multirow[t]{2}{*}{1} & Tumor a & $51,217,028$ & 17,083 & 24,932 & 665 & 55 & + & 110,548 & 112,177 \\
\hline & Normal b & $51,478,578$ & 17,010 & 24,521 & 624 & 33 & - & 85,635 & 112,710 \\
\hline \multirow[t]{2}{*}{2} & Tumor c & $51,098,090$ & 17,278 & 25,427 & 789 & 42 & - & 108,219 & 120,160 \\
\hline & Normal d & $51,157,672$ & 16,901 & 24,108 & 540 & 34 & - & 85,393 & 100,871 \\
\hline \multirow[t]{2}{*}{3} & Tumor e & $50,846,034$ & 18,089 & 26,379 & 1194 & 32 & + & 105,866 & 134,840 \\
\hline & Normal f & $51,363,004$ & 17,590 & 25,339 & 1005 & 26 & - & 103,312 & 164,495 \\
\hline
\end{tabular}

frameshifted during the transcript fusion process (Fig. 2).

\section{COL7A1-UCN2 CDNA validation}

In the 20 other tissue sample pairs, RT-PCR analysis revealed COL7A1-UCN2 cDNA expression in eleven of the LC samples but no TIC transcripts in the ANMMT samples (Fig. 3a). Thus, in this study of 23 LC patients, we detected COL7A1-UCN2 in 13 patients (57\%), and a comparison of the positive TIC distribution in the LC and ANMMT samples demonstrated that positive LC samples were statistically significantly more common than positive ANMMT samples $(p<0.0001)$ (Fig. $3 \mathrm{~b})$.

\section{Expression of COL7A1 and UCN2 mRNA}

Among all $23 \mathrm{LC}$ patients, the COL7A1: $\beta$-actin ratio in the ANMMT samples $(12.61 \pm 15.52)$ was significantly higher than that in the LC samples $(5.99 \pm 11.68 ; p=0.028)$
(Fig. 4a). Likewise, the $U C N 2: \beta$-actin ratio in the ANMMT samples $(17.02 \pm 21.69)$ was significantly higher than that in the LC samples $(7.34 \pm 14.90 ; p=0.021)$ (Fig. 4b). Furthermore, among all $23 \mathrm{LC}$ tissues, the COL7A1: $\beta$-actin ratio in the COL7A1-UCN2 TIC-positive samples $(3.89 \pm 8.56)$ was significantly lower than that in the COL7A1-UCN2 TICnegative samples (8.71 \pm 14.87 ; $p=0.019$ ) (Fig. 4c); likewise, the UCN2: $\beta$-actin ratio in COL7A1-UCN2 TIC-positive samples $(3.17 \pm 2.62)$ was also lower than that in the COL7A1-UCN2 TIC-negative samples $(12.84 \pm 21.85$; $p=0.21)$ (Fig. 4d).

\section{Disrupted coding regions of both COL7A1 and UCN2 in COL7A1-UCN2}

We compared the DNA sequences in the recurrent hybrid COL7A1 (rhCOL7A1, the sequence of COL7A1 in COL7A1-UCN2) and COL7A1. The rhCOL7A1 is located from exon 113 to exon 117 in a normal COL7A1 gene

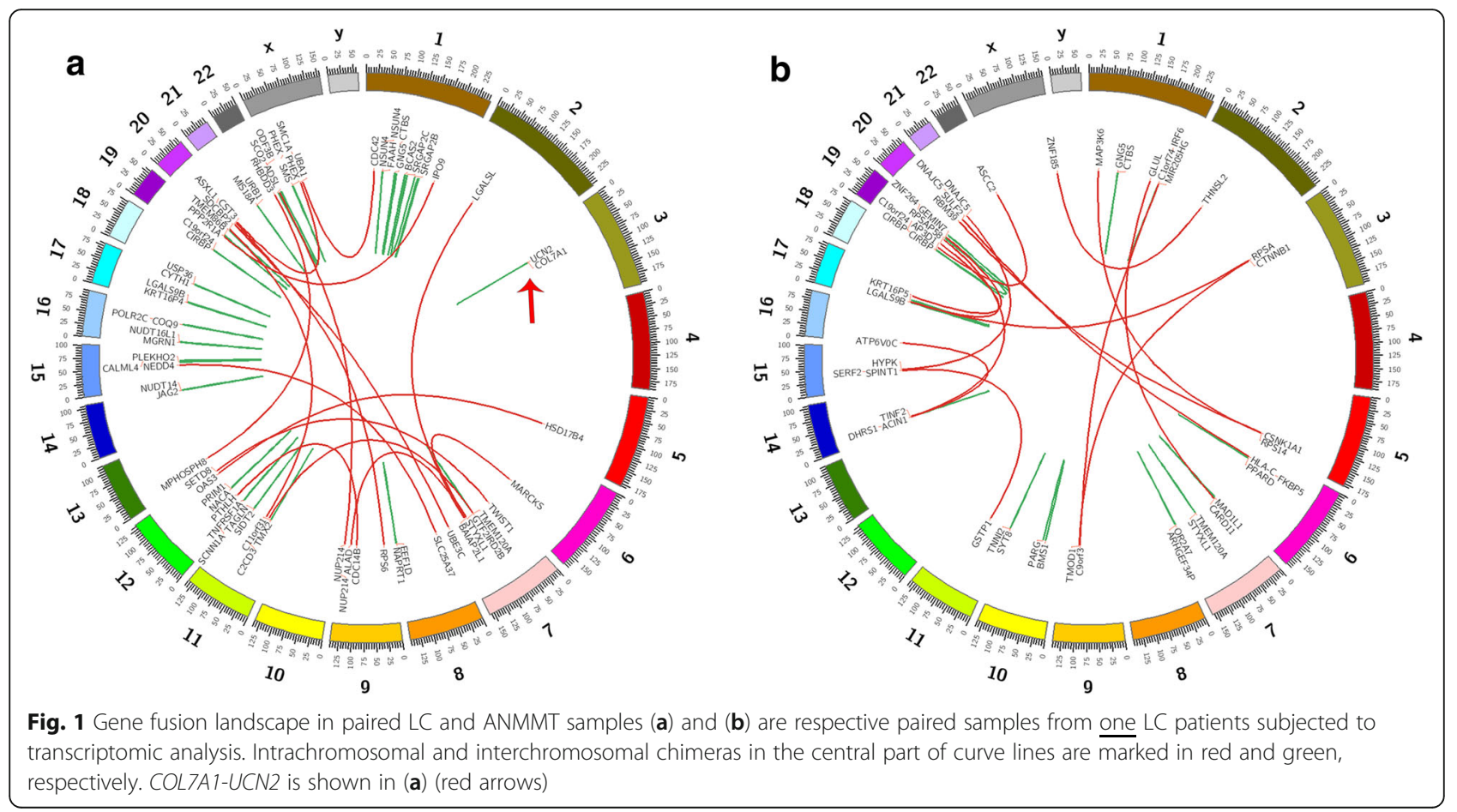


Table 4 Selected chimeras (10 out of 87 total) identified in three LC samples subjected to transcriptomic analysis

\begin{tabular}{|c|c|c|c|c|c|}
\hline Category & Up-stream Gene & Up-genome position & Down-stream Gene & Down-genome position & Frameshift \\
\hline Interchromosomal & ALAD & chr9: 116149551 & LAMTOR4 & chr7: 99751605 & NA \\
\hline Interchromosomal & ARHGAP8 & chr22: 45221411 & NUP214 & chr9: 134103680 & frame-shift \\
\hline Interchromosomal & ASXL1 & chr20: 31024580 & RPS6 & chr9: 19378798 & frame-shift \\
\hline Interchromosomal & BAIAP2L1 & chr7: 97984354 & MARCKS & chr6: 114180859 & frame-shift \\
\hline Interchromosomal & $\mathrm{C} 2 \mathrm{CD} 3$ & chr11: 73840431 & SMOX & chr20: 4101628 & NA \\
\hline Interchromosomal & CDC14B & chr9: 99284788 & PTHLH & chr12: 28116703 & frame-shift \\
\hline Interchromosomal & PPP2R1A & chr19: 52714563 & IPO9 & chr1: 201827608 & frame-shift \\
\hline Interchromosomal & CST3 & chr20: 23618257 & UBE3C & chr7: 157060279 & frame-shift \\
\hline Interchromosomal & HSD17B4 & chr5: 118809616 & OAS3 & chr12: 113410220 & NA \\
\hline Interchromosomal & $\mathrm{KMT} 2 \mathrm{E}$ & chr7: 104654982 & LGALSL & chr2: 64685419 & frame-shift \\
\hline Intrachromosomal & COL7A1 & chr3: 48602216 & UCN2 & chr3: 48600569 & frame-shift \\
\hline Intrachromosomal & COQ9 & chr16: 57494016 & POLR2C & chr16: 57496472 & frame-shift \\
\hline Intrachromosomal & DENND2C & chr1: 115128157 & BCAS2 & chr1: 115124012 & NA \\
\hline intrachromosomal & EEF1D & chr8: 144661949 & NAPRT1 & chr8: 144660113 & NA \\
\hline Intrachromosomal & GBP3 & chr1:89474630 & CCBL2 & chr1: 89435150 & frame-shift \\
\hline Intrachromosomal & HDGF & chr1: 156713444 & MRPL24 & chr1: 156711380 & frame-shift \\
\hline Intrachromosomal & KRT16P4 & chr17: 18354079 & LGALS9C & chr17:18387189 & NA \\
\hline Intrachromosomal & MAP2K5 & chr15: 68065090 & SKOR1 & chr15: 68118274 & frame-shift \\
\hline Intrachromosomal & MGRN1 & chr16: 4733933 & NUDT16L1 & chr16: 4744959 & frame-shift \\
\hline Intrachromosomal & NSUN4 & chr1: 46826500 & FAAH & chr1: 46867763 & frame-shift \\
\hline Intrachromosomal & NUDT14 & chr14: 105642871 & JAG2 & chr14: 105624100 & frame-shift \\
\hline Intrachromosomal & ODF3B & chr22: 50968332 & $\mathrm{SCO} 2$ & chr22: 50962852 & NA \\
\hline
\end{tabular}

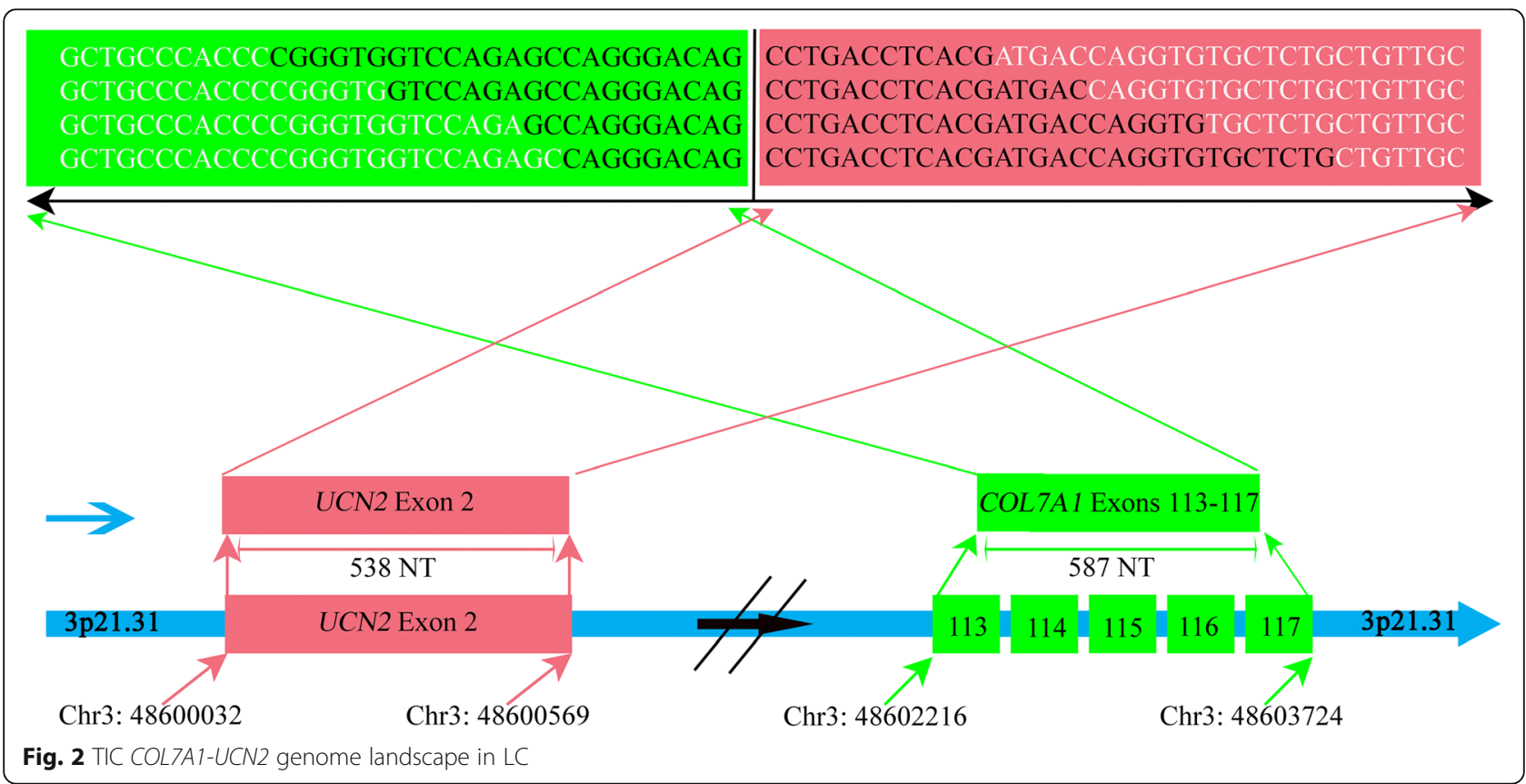




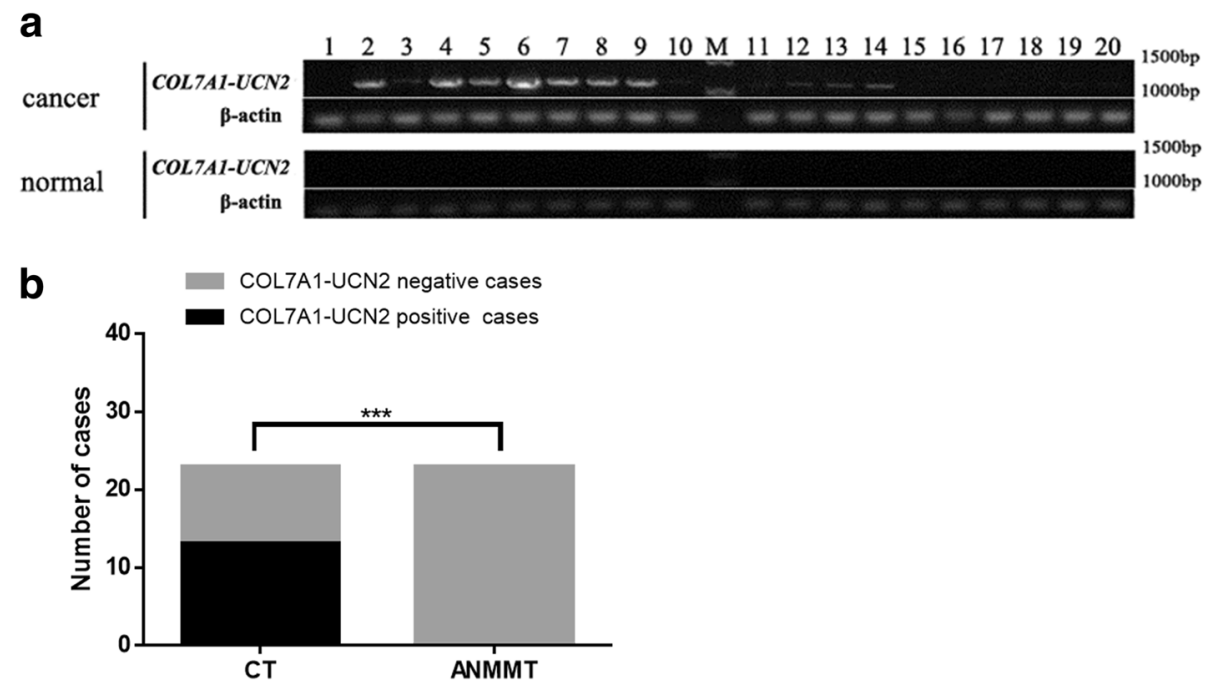

Fig. 3 RT-PCR validation of expression of TIC COLTAT-UCN2 CDNA in LC and ANMMT samples

(Fig. 5a). Besides, the DNA sequences in recurrent hybrid UCN2 (rhUCN2; the sequence of UCN2 in COL7A1-UCN2) and UCN2 were also compared. The rhUCN2 was composed of reversed nucleotides 1-540 of exon 2 in a normal UCN2 gene (Fig. 5b).

From the above, we found the COL7A1-UCN2 cDNA sequence and its predicted amino acid sequence, in which AG (highlighted in yellow) represents the last two nucleotides of COL7A1, which may translate into $\mathrm{S}$ (a serine amino acid, also highlighted in yellow), the first nucleotide of UCN2 (Fig. 6). Based on the above prediction, both the COL7A1 and UCN2 coding regions of COL7A1-UCN2 were disrupted.

Effect of COL7A1-UCN2 expression on overall survival in patients with LC

A Kaplan-Meier analysis revealed that LC patients who were positive for COL7A1-UCN2 had a significantly
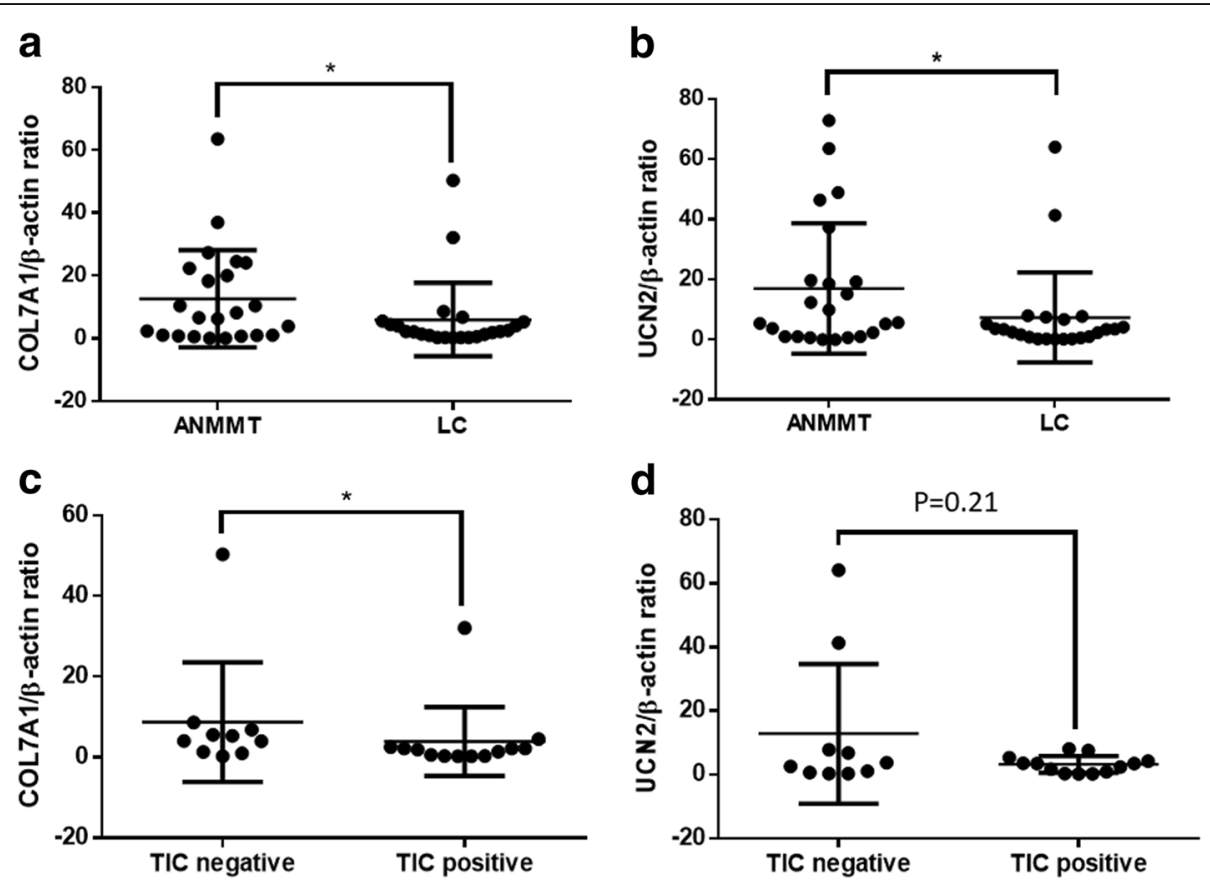

Fig. 4 Comparison of COL7A1 and UCN2 mRNA expression. LC, laryngeal cancer tissue; ANMMT, adjacent normal membrane mucous Tissue. (a) and (b) are comparison between LC and ANMMT samples; (c) and (d) are comparison between TIC COL7A1-UCN2 negativity and positivity LC samples. ${ }^{*} p<0.05$ 


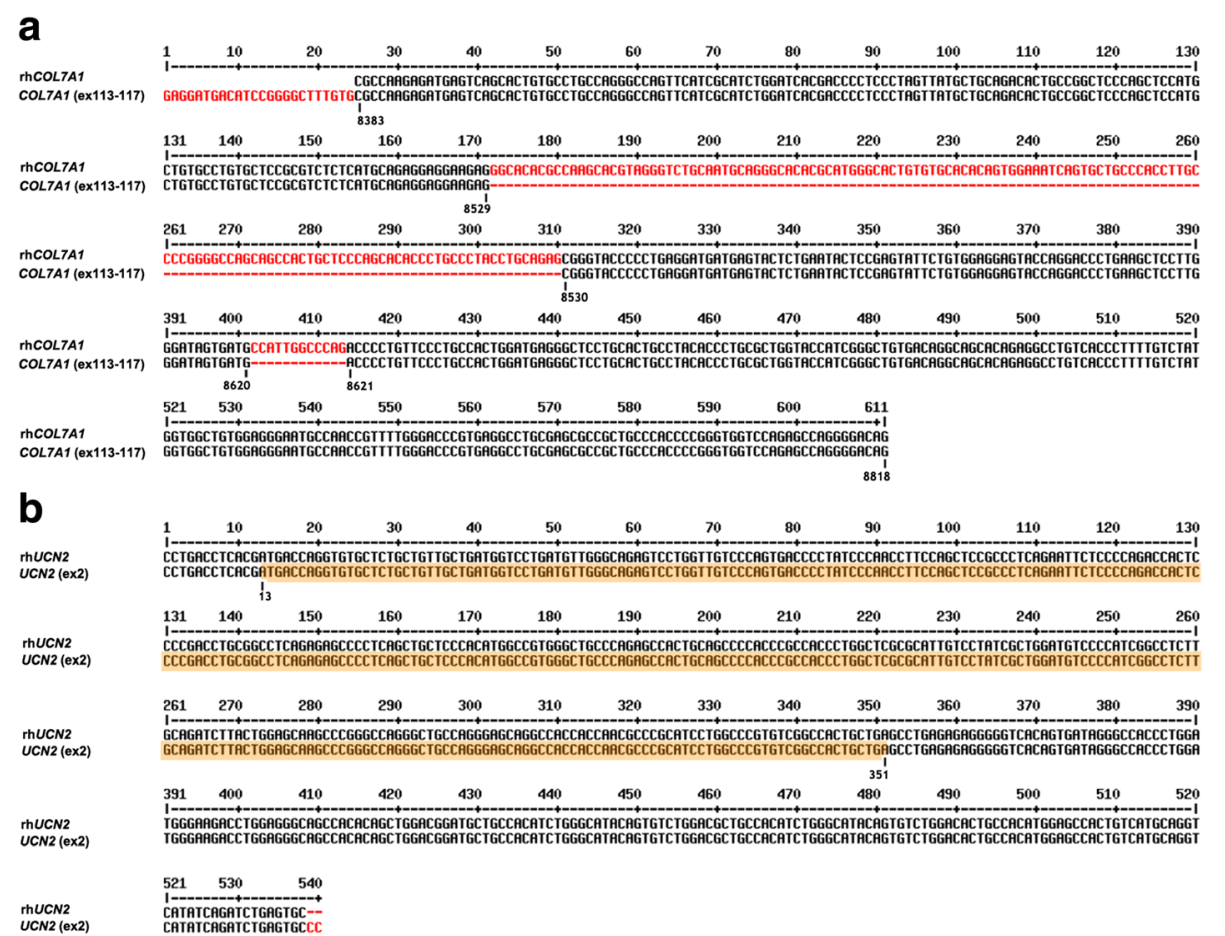

Fig. 5 Comparison of DNA sequences between recurrent-hybrid genes and normal genes. a Comparison of DNA sequences in recurrent-hybrid COL7A1 (rhCOL7A1; the sequence of COL7A1 in COL7A1-UCN2) and COL7A1 (from exon 113 to exon 117 in a normal COL7A1 gene). 8383, 8529, 8530, 8620,8621 , and 8818 are the gene sequence numbers in the encoding protein sequence. $\mathbf{b}$ Comparison of DNA sequences in recurrent-hybrid UCN2 (rhUCN2; the sequence of UCN2 in COL7A1-UCN2) and UCN2 (nucleotides 1-540 of exon 2 in a normal UCN2 gene). The gene sequences encoding for the final protein sequences are highlighted in orange. The consensus and inconsistent sequences are shown in black and red, respectively

worse overall survival time than those patients who were negative did ( $p=0.032$ [log-rank test]) (Fig. 7). Multivariable analysis demonstrated a significant association between COL7A1-UCN2 expression and overall survival (hazard ratio, 13.2 [95\% confidence interval, 1.2-149.5]).

\section{Discussion}

High-throughput transcriptome sequencing provides sufficient information with which to identify candidate oncogenic mRNA chimeras. These chimeric isoforms are usually generated by AS, which is a fundamental mechanism of transcript diversity generation [26-31]. AS generated the TIC COL7A1-UCN2 between neighboring genes, which is referred to as a read-through event [32]. In the present study, we found COL7A1UCN2 positivity in 13 of $23 \mathrm{LC}$ samples, whereas all 23 paired ANMMT samples were negative. This TIC was generated via alternative splicing in the cells of LC tissues. Furthermore, those LC tissues with COL7A1$U C N 2$ positivity had lower levels of COL7A1 and UCN2 mRNAs as compared to negative LC tissues. Therefore, this TIC potentially down-regulated the expression of the COL7A1 and UCN2 genes during and after chimera fusion; and it is thereby associated with poor clinical prognosis because both COL7A1 and UCN2 possessed explicit suppressor roles in tumor EMT regulation.

In a previous study, low or nonexistent COL7A1 expression was associated with the loss of the membrane basement, a specific extracellular matrix (ECM) component, and the promotion of the EMT process in cutaneous squamous cells (CSCCs) [33]. COL7A1-produced type VII collagen (ColVII) is the primary component of anchoring fibril protein, which constructs the membrane basement that separates the epithelium from the stroma in epithelial and mucous cells. Invasive epithelialmucous tumors can be distinguished from benign and pre-invasive lesions by the consistent loss of the surrounding linear basement membrane in a wide variety tissues [33-39]. The breakdown of the basement membrane is a critical early step in EMT, in which oncogenic derivatives of epithelial stem cells are thought to act as intrinsic cancer stem cells that disrupt the basement membrane via the secretion of matrix metalloproteinases (MMPs) [33]. In CSCCs, tumor cells with COL7A1 knockdown manifested increased migration and higher invasiveness, accompanied by the alteration of EMT marker expression (the decreased expression of E-cadherin and the increased expression of MMP2 and vimentin). Furthermore, ColVII knockdown can decrease 


\begin{abstract}
The cDNA sequence of COL7A1-UCN2 chimera
CGCCAAGAGATGAGTCAGCACTGTGCCTGCCAGGGCCAGTTCATCGCATCTGGATCACGACCCCTCCCTAGT TATGCTGCAGACACTGCCGGCTCCCAGCTCCATGCTGTGCCTGTGCTCCGCGTCTCTCATGCAGAGGAGGAA GAGGGCACACGCCAAGCACGTAGGGTCTGCAATGCAGGGCACACGCATGGGCACTGTGTGCACACAGTG GAAATCAGTGCTGCCCACCTTGCCCCGGGGCCAGCAGCCACTGCTCCCAGCACACCCTGCCCTACCTGCAG AGCGGGTACCCCCTGAGGATGATGAGTACTCTGAATACTCCGAGTATTCTGTGGAGGAGTACCAGGACCCTG AAGCTCCTTGGGATAGTGATGCCATTGGCCCAGACCCCTGTTCCCTGCCACTGGATGAGGGCTCCTGCACTG CCTACACCCTGCGCTGGTACCATCGGGCTGTGACAGGCAGCACAGAGGCCTGTCACCCTTTTGTCTATGGTG GCTGTGGAGGGAATGCCAACCGTTTTGGGACCCGTGAGGCCTGCGAGCGCCGCTGCCCACCCCGGGTGGT CCAGAGCCAGGGGACAG

CCTGACCTCACGATGACCAGGTGTGCTCTGCTGTTGCTGATGGTCCTGATGTTGGGCAGAGTCCTGGTTGTC CCAGTGACCCCTATCCCAACCTTCCAGCTCCGCCCTCAGAATTCTCCCCAGACCACTCCCCGACCTGCGGCCT CAGAGAGCCCCTCAGCTGCTCCCACATGGCCGTGGGCTGCCCAGAGCCACTGCAGCCCCACCCGCCACCCT GGCTCGCGCATTGTCCTATCGCTGGATGTCCCCATCGGCCTCTTGCAGATCTTACTGGAGCAAGCCCGGGCC AGGGCTGCCAGGGAGCAGGCCACCACCAACGCCCGCATCCTGGCCCGTGTCGGCCACTGCTGAGCCTGAG AGAGGGGGTCACAGTGATAGGGCCACCCTGGATGGGAAGACCTGGAGGGCAGCCACACAGCTGGACGGA TGCTGCCACATCTGGGCATACAGTGTCTGGACGCTGCCACATCTGGGCATACAGTGTCTGGACACTGCCACA TGGAGCCACTGTCATGCAGGTCATATCAGATCTGAGTGC (1125 nts)

Predicted amino acid sequence

RQEMSQHCACQGQFIASGSRPLPSYAADTAGSQLHAVPVLRVSHAEEEEGTRQARRVCNAGHTHGHCVHTV EISAAHLAPGPAATAPSTPCPTCRAGTP-G--VL-ILRVFCGGVPGP-SSLG--CHWPRPLFPATG-GLLHCLHPALVP SGCDRQHRGLSPFCLWWLWRECQPFWDP-GLRAPLPTPGGPEPGDSLTSR-PGVLCCC-WS-CWAESWLSQPLSQPSSSALRILPRPLPDLRPQRAPQLLPHGRGLPRATAAPPATLARALSYRWMSPSASCRSYWSKPGPGLPGS RPPPTPASWPVSATAEPERGGHSDRATLDGKTWRAATQLDGCCHIWAYSVWTLPHLGIQCLDTATWSHCHAG HIRSEC
\end{abstract}

Fig. 6 The COL7AT-UCN2 CDNA sequence and its predicted amino acid sequence. AG (highlighted in yellow) represents the last two nucleotides of COL7A1, which may translate into S (Serine, also highlighted in yellow) with the first nucleotide of UCN2

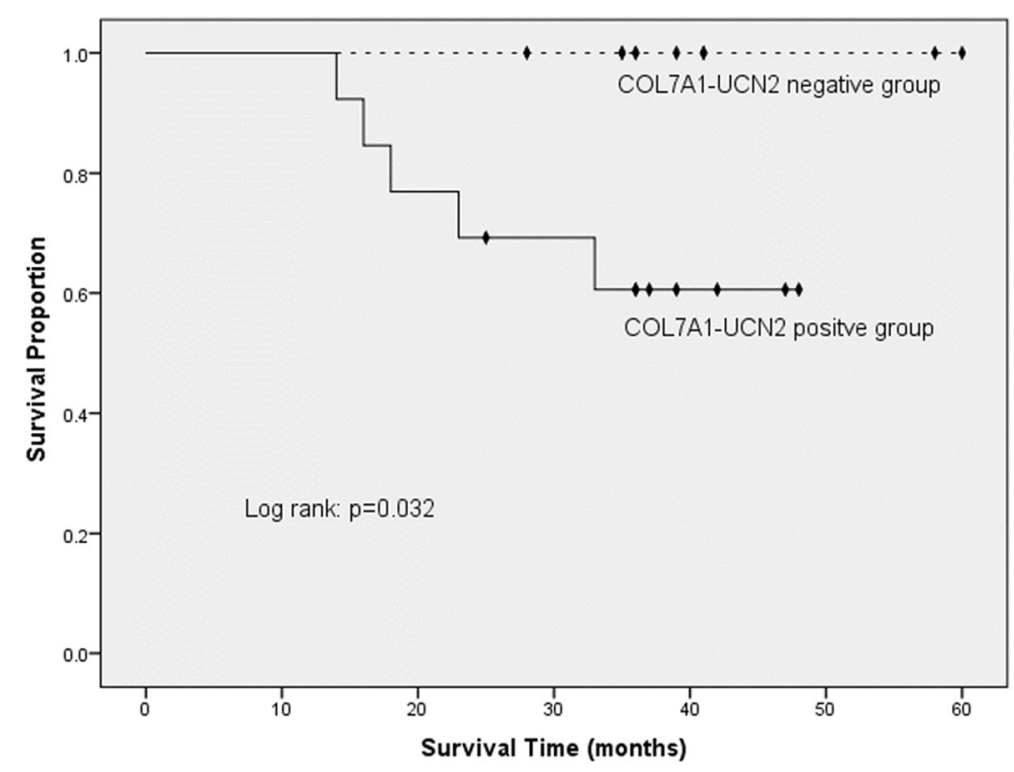

Fig. 7 Effect of COLTA7-UCN2 expression on overall survival in patients positive and negative for this TIC 
epithelial cancer cell differentiation and increase the expression of the chemokine ligand receptors CXCL10CXCR3 and PLC- $\beta 4$, which can further facilitate EMT and increase tumor invasion through an autocrine forward loop [22].

In our present study, COL7A1 mRNA levels were down-regulated in cancer tissues, and the COL7A1UCN2 chimera generation mechanism circumvented TGF- $\beta 1$ 's tumor-suppressive effects and thereby promoted tumor invasion and proliferation. TGF- $\beta 1$ maintained normal tissue homeostasis and could both suppress and promote tumor proliferation in a timeand concentration-dependent manner $[20,40,41]$. Within this homeostasis, TGF- $\beta 1$ broadly controlled the ECM, providing transcription regulation for the following genes: COL1A1, COL1A2, COL3A1, COL5A2, COL6A1, COL6A3, COL7A1, etc. The ECM is a dense latticework of collagen and elastin that serves as a selective macromolecular filter, it plays a role in mitogenesis and differentiation [42, 43]. Therefore, abnormal ECM homeostasis is a hallmark of cancer. It may be associated with the dysregulation of various collagens and increased tumor invasion because COL7A1-produced collagen VII is an essential component of various collagens $[20,43]$. TGF- $\beta 1$ can up-regulate collagen VII in tissues given normal homeostasis, a high concentration, and longterm exposure to TGF- $\beta 1$ [42]. Collagen VII was found to be down-regulated in cancer tissues, and homeostasis was lost through epigenetic transcription regulation [44], canonical pathway inactivation in TGF- $\beta 1$ (i.e., TGFR mutation) in cancer cells [45], or ECM alteration in the tumor microenvironment [46]. In our study, we found that cancer tissues had significantly decreased COL7A1 mRNA levels as compared to paired normal tissues, and we also found that cancer tissues with COL7A1-UCN2 chimera positivity had significantly lower COL7A1 mRNA levels than the cancer tissues with COL7A1$U C N 2$ chimera negativity. These results might support that the COL7A1-UCN2 chimera generation mechanism may be associated with the down-regulation of COL7A1 mRNA, which is reflected the degree of invasiveness found in tumor cells.

The activation of the UCN2/corticotropin-releasing factor receptor 2 (CRFR2) axis signaling can inhibit tumor vascularization, cell proliferation and invasion, and EMT [21, 47], whereas the mechanism of COL7A1$U C N 2$ chimera generation can potentially down-regulate UCN2 mRNA and thereby cause the loss of its tumor suppressor role. Both UCN2 and CRFR2 belong to the $\mathrm{CRH}$ family, which is known to contain the principal neuroendocrine regulators of stress response in the central nervous system [21, 47, 48]. However, previous studies found that the dysregulation of UCN2/CRFR2 signaling was associated with prostate cancer [49], non- small cell lung carcinoma [50], colorectal cancer (CRC) [21], Lewis lung carcinoma (LLC) [47], and human adrenal and ovarian tumors [51]. Specifically, in vivo and in vitro studies found that UCN2/CRFR2 activation inhibited tumor vascularization and cell proliferation and invasion [21, 47]. Furthermore, in CRC cell lines, the blockage of the UCN2/CRFR2 axis promoted EMT (the altered expression of EMT marker, decreased vimentin, and increased E-cadherin and glycogen synthase kinase $3 \beta$ expression) via persistent interleukin-6/ Stat3 signaling (colonic inflammation regulation) [21].

The coding regions of both COL7A1 and UCN2 were disrupted or destroyed in COL7A1-UCN2, and this TIC did not encode a fusion protein. COL7A1 protein includes a Kunitz domain, the deactivation of which induces tumorigenesis [52]. In the rhCOL7A1 coding region, the Kunitz domain is the first 49 residues in the predicted amino acid sequence of COL7A1-UCN2, whereas the remaining 96 residues of the Kunitz domain may be disrupted by $U C N 2$ sequence insertion. In the rh $U C N 2$ coding region, $U C N 2$ was frame-shifted, and a discontinuous sequence in the coding region may also disrupt normal UCN2 expression, although COL7A1UCN2 includes the complete nucleotides for encoding UCN2 (13-351 nt; 112 amino acids) (Figs. 5 and 6). Therefore, in line with the results of a previous study [14], COL7A1-UCN2 produced no fusion proteins or independent transcripts.

The presence of COL7A1-UCN2 in LCs was not the result of stochastic processes. Instead, it was a reflection of DNA damage to a severe degree, and thus it may be associated with poor prognosis. First, we found COL7A1-UCN2 positivity in 13 of 23 LC samples, whereas all 23 paired ANMMT samples were negative. Second, we found consistent, precise RNA junctions in every recurrent validation in all COL7A1-UCN2-positive patient samples. Third, highly expressed genes did not generate TICs randomly. Fourth, a Kaplan-Meier analysis revealed that patients who were positive for COL7A1-UCN2 had significantly worse overall survival times than did those who were negative.

This study had certain limitations. To validate the DNA rearrangements in chromosomes, the use of a standard fluorescence in situ hybridization (FISH) assay necessitated a minimum distance between the two fused genes $(100-150 \mathrm{~kb})$ [53], but the distance between the adjacent ends of COL7A1 and UCN2 is less than $20 \mathrm{~kb}$. Thus, we only used long-range RT-PCR to detect the occurrence of COL7A1-UCN2 cDNA expression in the LC samples. Also, in the AS events, whether the intrinsic TIC-generation mechanism occurs via cis-splicing or trans-splicing remains unknown [29]. Determining whether TICs function as noncoding RNAs or regulatory RNAs in cancer cell lines without protein participation 
requires further in vitro evidence. Finally, although our patient sample size was small and potential selection bias could exist, our findings on COL7A1-UCN2 TIC may provide some novel information to help generate new hypothesis for our future study.

\section{Conclusion}

Our results indicated that the TIC COL7A1-UCN2 is highly common and enriched in LC samples and that its expression may be associated with LC-cell transition, EMT promotion, and poor LC prognosis. Although its intrinsic generation mechanisms remain largely unknown, COL7A1-UCN2 may serve as a diagnostic biomarker for early the detection of $\mathrm{LC}$, as well as $\mathrm{LC}$ prognosis.

\section{Additional file}

Additional file 1: Figure S1. Gene fusion landscape in the other 2 paired $L C$ and ANMMT samples. $\mathbf{c}$ and $\mathbf{d}$, and $\mathbf{e}$ and $\mathbf{f}$ are respective paired samples from the other $2 \mathrm{LC}$ patients subjected to transcriptomic analysis ( $\mathbf{a}$ and $\mathbf{b}$ are in Fig. 1). Intrachromosomal and interchromosomal chimeras in the central part of curve lines are marked in red and green, respectively. COL7A1-UCN2 is shown in e (red arrows). (TIFF $1477 \mathrm{~kb}$ )

\section{Abbreviations}

ANMMT: Adjacent normal mucous membrane tissue; AS: Alternative splicing; ColVII: Type VII collagen; CRC: Colorectal cancer; CRFR2: Corticotropinreleasing factor receptor 2; ECM: Extracellular matrix; EMT: Epithelialmesenchymal transition; FISH: Fluorescence in situ hybridization; LC: Laryngeal cancer; LLC: Lewis lung carcinoma; MMPs: Matrix metalloproteinases; RPKM: Reads per kilobase exon model per million reads; RT-PCR: Real-time polymerase chain reaction; TIC: Transcription-induced chimera; UCN2: Urocortin 2

\section{Acknowledgments}

None.

\section{Availability of data materials}

The datasets used and/or analyzed during the current study are available from the corresponding author on reasonable request.

\section{Funding}

This work was supported by China National Science Foundation (Grant N0.81670946), which supported the design of the study and collection, analysis, and interpretation of data and in writing the manuscript. The funding body had no role in the design of the study and collection, analysis, and interpretation of data and in writing the manuscript.

\section{Authors' contributions}

$Y T, X F, J Y, Y Z$ and $Z H$ carried out the majority of the experiment, data analysis, and wrote the manuscript. $Y T, X F, N G, J Y, M T, X L, G L, Y Z$, and $Z H$ made substantial contributions to conception and design, or acquisition of data, or analysis and interpretation of data. All authors reviewed and approved the final manuscript.

\section{Ethics approval and consent to participate}

The study protocol was approved by the Ethics Committee of the Beijing Tong Hospital, Capital Medical University (No. TRECKY 2009-33; Date: Jan, 2009). The consent form was obtained from each study patients.

\section{Consent for publication}

All patients provided their written informed consent for their data and tissues for the study. This manuscript contains individual person's data, for which consent to publish were obtained from that person.

\section{Competing interests}

The authors declare that they have no competing interests.

\section{Publisher's Note}

Springer Nature remains neutral with regard to jurisdictional claims in published maps and institutional affiliations.

\section{Author details}

${ }^{1}$ Department of Otolaryngology-Head and Neck Surgery, Key Laboratory of Otolaryngology Head and Neck Surgery, Beijing Tongren Hospital, Capital Medical University, Beijing 100730, China. ${ }^{2}$ Department of Head and Neck Surgery, The University of Texas MD Anderson Cancer Center, Houston, TX 77030, USA. ${ }^{3}$ Hefei National Laboratory for Physical Sciences at Microscale, Innovation Centre for Cell Signaling Network, School of Life Science, University of Science and Technology of China, Hefei, Anhui 230026, People's Republic of China. ${ }^{4}$ Department of Otolaryngology-Head and Neck Surgery, the Second Affiliated Hospital of Anhui Medical University, Hefei 230601, China.

Received: 8 August 2017 Accepted: 21 February 2018

Published online: 02 March 2018

\section{References}

1. Chen W, Zheng R, Baade PD, Zhang S, Zeng H, Bray F, Jemal A, Yu XQ, He J. Cancer statistics in China, 2015. CA Cancer J Clin. 2016;66(2):115-32.

2. Teyssier JR. The chromosomal analysis of human solid tumors a triple challenge. Cancer Genet Cytogenet. 1989;37(1):103-25.

3. Brugere J, Guenel P, Leclerc A, Rodriguez J. Differential effects of tobacco and alcohol in cancer of the larynx, pharynx, and mouth. Cancer. 1986;57(2):391-5.

4. Incze J, Vaughan CW, Lui P, Strong MS, Kulapaditharom B. Premalignant changes in normal appearing epithelium in patients with squamous cell carcinoma of the upper aerodigestive tract. Am J Surg. 1982;144(4):401-5.

5. Shin DM, Kim J, Ro JY, Hittelman J, Roth JA, Hong WK, Hittelman WN. Activation of $p 53$ gene expression in premalignant lesions during head and neck tumorigenesis. Cancer Res. 1994:54(2):321-6.

6. Soda M, Choi YL, Enomoto M, Takada S, Yamashita Y, Ishikawa S, Fujiwara SI, Watanabe $\mathrm{H}$, Kurashina $\mathrm{K}$, Hatanaka $\mathrm{H}$. Identification of the transforming EML4-ALK fusion gene in non-small-cell lung cancer. Nature. 2007; 448(7153):561-6.

7. Mitelman F, Johansson B, Mertens F. The impact of translocations and gene fusions on cancer causation. Nat Rev Cancer. 2007;7(4):233-45.

8. Mertens F, Antonescu CR, Mitelman F. Gene fusions in soft tissue tumors: recurrent and overlapping pathogenetic themes. Genes Chromosomes Cancer. 2016:55(4):291-310.

9. Dave SS, Fu K, Wright GW, Lam LT, Kluin P, Boerma E-J, Greiner TC, Weisenburger DD, Rosenwald A, Ott G. Molecular diagnosis of Burkitt's lymphoma. N Engl J Med. 2006;354(23):2431-42.

10. Sidhar SK, Clark J, Gill S, Hamoudi R, Crew AJ, Gwilliam R, Ross M, Linehan WM, Birdsall S, Shipley J. The t $\left(X_{;}\right.$1)(p11. 2; q21.2) translocation in papillary renal cell carcinoma fuses a novel gene PRCC to the TFE3 transcription factor gene. Hum Mol Genet. 1996;5(9):1333-8.

11. Kroll TG, Sarraf P, Pecciarini L, Chen C-J, Mueller E, Spiegelman BM, Fletcher JA. PAX8-PPARY1 fusion in oncogene human thyroid carcinoma. Science. 2000;289(5483):1357-60

12. Panagopoulos I, Tiziana Storlazzi C, Fletcher CD, Fletcher JA, Nascimento A, Domanski HA, Wejde J, Brosjö O, Rydholm A, Isaksson M. The chimeric FUS/ CREB312 gene is specific for low-grade fibromyxoid sarcoma. Genes Chromosom Cancer. 2004;40(3):218-28.

13. Tomlins SA, Rhodes DR, Perner S, Dhanasekaran SM, Mehra R, Sun X-W, Varambally S, Cao X, Tchinda J, Kuefer R. Recurrent fusion of TMPRSS2 and ETS transcription factor genes in prostate cancer. Science. 2005; 310(5748):644-8.

14. Parra G, Reymond A, Dabbouseh N, Dermitzakis ET, Castelo R, Thomson TM, Antonarakis SE, Guigo R. Tandem chimerism as a means to increase protein complexity in the human genome. Genome Res. 2006;16(1):37-44.

15. Li H, Wang J, Mor G, Sklar J. A neoplastic gene fusion mimics trans-splicing of RNAs in normal human cells. Science. 2008;321(5894):1357-61.

16. Iyer MK, Chinnaiyan AM, Maher CA. ChimeraScan: a tool for identifying chimeric transcription in sequencing data. Bioinformatics. 2011;27(20):2903-4. 
17. McPherson A, Hormozdiari F, Zayed A, Giuliany R, Ha G, Sun MG, Griffith M, Heravi Moussavi A, Senz J, Melnyk N, et al. deFuse: an algorithm for gene fusion discovery in tumor RNA-Seq data. PLoS Comput Biol. 2011;7(5):e1001138.

18. Lau C-C, Sun T, Ching AK, He M, Li J-W, Wong AM, Co NN, Chan AW, Li P-S, Lung RW. Viral-human chimeric transcript predisposes risk to liver cancer development and progression. Cancer Cell. 2014;25(3):335-49.

19. Zhang Y, Gong M, Yuan H, Park HG, Frierson HF, Li H. Chimeric transcript generated by cis-splicing of adjacent genes regulates prostate cancer cell proliferation. Cancer Discov. 2012;2(7):598-607.

20. Martins VL, Caley MP, Moore K, Szentpetery Z, Marsh ST, Murrell DF, Kim MH, Avari M, McGrath JA, Cerio R, et al. Suppression of TGF beta and Angiogenesis by type VII collagen in cutaneous SCC. J Natl Cancer Inst. 2016;108(1)

21. Rodriguez JA, Huerta-Yepez S, Law IK, Baay-Guzman GJ, Tirado-Rodriguez B, Hoffman JM, Iliopoulos D, Hommes DW, Verspaget HW, Chang L, et al. Diminished expression of CRHR2 in human colon cancer promotes tumor growth and EMT via persistent IL-6/Stat3 signaling. Cell Mol Gastroenterol Hepatol. 2015;1(6):610-30.

22. Martins VL, Vyas JJ, Chen M, Purdie K, Mein CA, South AP, Storey A, McGrath JA, O'Toole EA. Increased invasive behaviour in cutaneous squamous cell. carcinoma with loss of basement-membrane type VII collagen. J Cell Sci. 2009;122(11):1788-99.

23. Furusaka T, Matuda H, Saito T, Katsura Y, Ikeda M. Long-term observations and salvage operations on patients with T2NOMO squamous cell carcinoma of the glottic larynx treated with radiation therapy alone. Acta Otolaryngol. 2012;132(5):546-51.

24. Jia W, Qiu K, He M, Song P, Zhou Q, Zhou F, Yu Y, Zhu D, Nickerson ML, Wan S. SOAPfuse: an algorithm for identifying fusion transcripts from paired-end RNA-Seq data. Genome Biol. 2013;14(2):R12.

25. Ge H, Liu K, Juan T, Fang F, Newman M, Hoeck W. FusionMap: detecting fusion genes from next-generation sequencing data at base-pair resolution. Bioinformatics. 2011;27(14):1922-8.

26. Edgren $\mathrm{H}$, Murumagi A, Kangaspeska S, Nicorici D, Hongisto V, Kleivi K, Rye $\mathbb{H}_{\text {, }}$ Nyberg S, Wolf M, Borresen-Dale AL, et al. Identification of fusion genes in breast cancer by paired-end RNA-sequencing. Genome Biol. 2011;12(1):R6.

27. Sbone A: FusionSeq: a modular framework for finding gene fusions by analyzing paired-end 2010

28. Wang K, Singh D, Zeng Z, Coleman SJ, Huang Y, Savich GL, He X, Mieczkowski P, Grimm SA, Perou CM, et al. MapSplice: accurate mapping of RNA-seq reads for splice junction discovery. Nucleic Acids Res. 2010;38(18):e178

29. Kannan K, Wang L, Wang J, Ittmann MM, Li W, Yen L. Recurrent chimeric RNAs enriched in human prostate cancer identified by deep sequencing Proc Natl Acad Sci U S A. 2011;108(22):9172-7.

30. Zhao Q, Caballero OL, Levy S, Stevenson BJ, Iseli C, de Souza SJ, Galante PA, Busam D, Leversha MA, Chadalavada K, et al. Transcriptome-guided characterization of genomic rearrangements in a breast cancer cell line. Proc Natl Acad Sci U S A. 2009;106(6):1886-91.

31. Gingeras TR. Implications of chimaeric non-co-linear transcripts. Nature. 2009;461(7261):206-11.

32. Maher CA, Kumar-Sinha C, Cao X, Kalyana-Sundaram S, Han B, Jing X, Sam L, Barrette T, Palanisamy N, Chinnaiyan AM. Transcriptome sequencing to detect gene fusions in cancer. Nature. 2009;458(7234):97-101.

33. Horejs CM. Basement membrane fragments in the context of the epithelialto-mesenchymal transition. Eur J Cell Biol. 2016;95:427-40.

34. Barsky SH, Rao NC, Restrepo C, Liotta LA. Immunocytochemical enhancement of basement membrane antigens by pepsin: applications in diagnostic pathology. Am J Clin Pathol. 1984;82(2):191-4.

35. Birembaut $P$, Caron $Y$, Adnet JJ, Foidart JM. Usefulness of basement membrane markers in tumoural pathology. J Pathol. 1985;145(4):283-96.

36. Gelse K. Collagens - structure, function, and biosynthesis. Adv Drug Deliv Rev. 2003:55(12):1531-46

37. Pozzi A, Yurchenco PD, lozzo RV. The nature and biology of basement membranes. Matrix Biol. 2017;57-58:1-11.

38. Uitto J, Christiano AM. Molecular genetics of the cutaneous basement membrane zone. Perspectives on epidermolysis bullosa and other blistering skin diseases. J Clin Invest. 1992;90(3):687-92.

39. Uitto J, Pulkkinen L. Molecular complexity of the cutaneous basement membrane zone. Mol Biol Rep. 1996;23(1):35-46.

40. Fuxe J, Vincent T, Garcia de Herreros A. Transcriptional crosstalk between TGF-beta and stem cell pathways in tumor cell invasion: role of EMT promoting Smad complexes. Cell Cycle. 2010;9(12):2363-74.
41. Knaup J, Gruber C, Krammer B, Ziegler V, Bauer J, Verwanger T. TGF betasignaling in squamous cell carcinoma occurring in recessive dystrophic epidermolysis bullosa. Anal Cell Pathol. 2011;34(6):339-53.

42. Vindevoghel L, Kon A, Lechleider RJ, Uitto J, Roberts AB, Mauviel A. Smaddependent transcriptional activation of human type VII collagen gene (COL7A1) promoter by transforming growth factor-beta. J Biol Chem. 1998; 273(21):13053-7.

43. Verrecchia F, Chu M-L, Mauviel A. Identification of novel TGF- $3 /$ Smad gene targets in dermal fibroblasts using a combined CDNA microarray/promoter transactivation approach. J Biol Chem. 2001;276(20):17058-62.

44. Chernov AV, Strongin AY. Epigenetic regulation of matrix metalloproteinases and their collagen substrates in cancer. Biomol Concepts. 2011;2(3):135-47.

45. Massagué J. TGF $\beta$ in cancer. Cell. 2008;134(2):215-30.

46. Kessenbrock K, Plaks V, Werb Z. Matrix metalloproteinases: regulators of the tumor microenvironment. Cell. 2010;141(1):52-67.

47. Hao Z, Huang Y, Cleman J, Jovin IS, Vale WW, Bale TL, Giordano FJ. Urocortin2 inhibits tumor growth via effects on vascularization and cell proliferation. Proc Natl Acad Sci U S A. 2008;105(10):3939-44.

48. Reubi JC, Waser B, Vale W, Rivier J. Expression of CRF1 and CRF2 receptors in human cancers. J Clin Endocrinol Metab. 2003;88(7):3312-20.

49. Tezval H, Jurk S, Atschekzei F, Serth J, Kuczyk MA, Merseburger AS. The involvement of altered corticotropin releasing factor receptor 2 expression in prostate cancer due to alteration of anti-angiogenic signaling pathways. Prostate. 2009;69(4):443-8.

50. Wang J, Jin L, Chen J, Li S. Activation of corticotropin-releasing factor receptor 2 inhibits the growth of human small cell lung carcinoma cells. Cancer Investig. 2009;28(2):146-55.

51. Suda T, Tomori N, Yajima F, Odagiri E, Demura H, Shizume K. Characterization of immunoreactive corticotropin and corticotropinreleasing factor in human adrenal and ovarian tumours. Acta Endocrinol. 1986;111(4):546-52.

52. Ranasinghe S, McManus DP. Structure and function of invertebrate Kunitz serine protease inhibitors. Dev Comp Immunol. 2013;39(3):219-27.

53. Rickman DS, Pflueger D, Moss B, VanDoren VE, Chen CX, de la Taille A Kuefer R, Tewari AK, Setlur SR, Demichelis F. SLC45A3-ELK4 is a novel and frequent erythroblast transformation-specific fusion transcript in prostate cancer. Cancer Res. 2009;69(7):2734-8.

\section{Submit your next manuscript to BioMed Central and we will help you at every step:}

- We accept pre-submission inquiries

- Our selector tool helps you to find the most relevant journal

- We provide round the clock customer support

- Convenient online submission

- Thorough peer review

- Inclusion in PubMed and all major indexing services

- Maximum visibility for your research

Submit your manuscript at www.biomedcentral.com/submit 